University of Wollongong

Research Online

Faculty of Informatics - Papers (Archive)

Faculty of Engineering and Information

Sciences

18-10-2006

\title{
Ultra-wideband MB-OFDM channel estimation with complementary codes
}

Darryn Lowe

University of Wollongong, darrynl@uow.edu.au

Xiaojing Huang

University of Wollongong, huang@uow.edu.au

Follow this and additional works at: https://ro.uow.edu.au/infopapers

Part of the Physical Sciences and Mathematics Commons

\section{Recommended Citation}

Lowe, Darryn and Huang, Xiaojing: Ultra-wideband MB-OFDM channel estimation with complementary codes 2006.

https://ro.uow.edu.au/infopapers/407

Research Online is the open access institutional repository for the University of Wollongong. For further information contact the UOW Library: research-pubs@uow.edu.au 


\title{
Ultra-wideband MB-OFDM channel estimation with complementary codes
}

\author{
Abstract \\ In this paper, we design complementary codesets that significantly improve the quality of channel \\ estimation in orthogonal frequency division multiplexing (OFDM) communication systems, with a focus \\ on the recent MB-OFDM ultra-wideband (UWB) standard. The proposed time-domain OFDM channel \\ estimation technique incurs only a nominal increase in computational complexity and is able to be readily \\ retrofitted into the existing MB-OFDM standard. The underlying complementary codesets, found via an \\ evolutionary algorithm, combine with the existing preamble synchronization sequences to yield \\ asymptotically ideal auto-correlation functions (ACFs). We show how improvements exceeding $1 \mathrm{~dB}$ can \\ be achieved in end-to-end packet error rate relative to conventional zero-forcing OFDM equalization.

\section{Keywords} \\ UWB, ultra-wideband, MB-OFDM, equalization, complementary, estimation \\ Disciplines \\ Physical Sciences and Mathematics

\section{Publication Details} \\ This article was originally published as: Lowe, D \& Huang, X, Ultra-wideband MB-OFDM channel \\ estimation with complementary codes, 2006 International Symposium on Communications and \\ Information Technologies (ISCIT), Bangkok, Thailand, 18-20 October 2006. Conference information is \\ available here.
}




\title{
Ultra-Wideband MB-OFDM Channel Estimation with Complementary Codes
}

\author{
Darryn Lowe and Xiaojing Huang \\ School of Electrical, Computer and Telecommunications Engineering \\ University of Wollongong \\ Wollongong, Australia, 2522 \\ Email: \{darrynl, huang\}@uow.edu.au
}

\begin{abstract}
In this paper, we design complementary codesets that significantly improve the quality of channel estimation in orthogonal frequency division multiplexing (OFDM) communication systems, with a focus on the recent MB-OFDM ultrawideband (UWB) standard. The proposed time-domain OFDM channel estimation technique incurs only a nominal increase in computational complexity and is able to be readily retrofitted into the existing MB-OFDM standard. The underlying complementary codesets, found via an evolutionary algorithm, combine with the existing preamble synchronization sequences to yield asymptotically ideal auto-correlation functions (ACFs). We show how improvements exceeding $1 \mathrm{~dB}$ can be achieved in end-to-end packet error rate relative to conventional zero-forcing OFDM equalization.
\end{abstract}

\section{INTRODUCTION}

Coherent signalling over frequency-selective channels requires that the receiver estimate and equalize the channel prior to demodulation. To that end, one of the most significant advantages of orthogonal frequency division multiplexing (OFDM) systems [1], typified by the block diagram of Fig. 1 , is that the highlighted channel estimation and equalization stage is conceptually and computationally simple. Since this allows an OFDM transceiver to be both low-power and lowcost, OFDM systems are becoming increasingly popular in applications ranging from wireless personal area networks (WPANs) to digital television.

The simplest OFDM channel estimation is a zero-forcing (ZF) approximation of $N$ complex coefficients to rotate and scale each of the $N$ subcarriers in the received OFDM symbol. Such channel estimation is most commonly performed using an explicit training sequence known to both sender and receiver. Although blind estimation [2] techniques exist, longer convergence times make them more appropriate for channel tracking than for initial channel estimation.

Most OFDM channel estimation sequences possess a flat power-spectral density so that each subcarrier can be estimated to the same accuracy. For example, in multi-band OFDM (MBOFDM) [3], the channel estimation sequence is obtained by taking the inverse fast fourier transform (IFFT) of $N$ equalmagnitude random quadrature phase shift keying (QPSK) symbol constellations. This allows the receiver to perform a ZF frequency-domain equalization (FDE) at the cost of a single fast fourier transform (FFT) and $N$ complex multiplications. Unlike many iterative time-domain equalization (TDE)

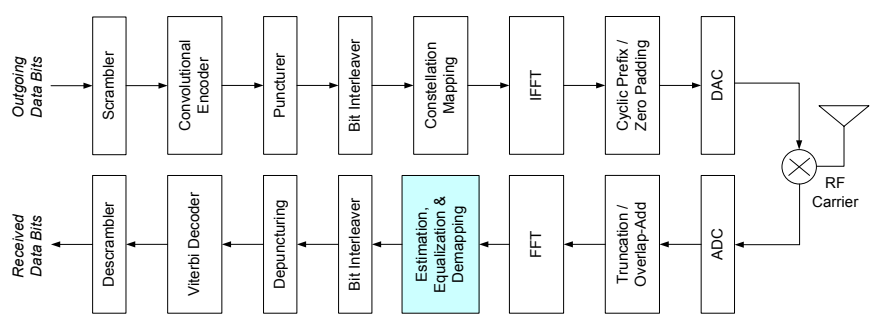

Fig. 1. Block diagram of an generic OFDM transmitter.

techniques, ZF FDE is guaranteed to converge and is low in computational complexity.

In this paper, we present an alternative estimation and equalization scheme that reuses the correlation of the synchronization sequence. In other words, we derive a channel estimation sequence that, while possessing a non-ideal autocorrelation function (ACF) in-and-of-itself, can be combined with an existing preamble synchronization sequence to create a complementary codeset with an asymptotically ideal ACF. As the receiver hardware must already implement a correlator for packet detection, this approach to channel estimation requires only nominal additional logic. Once the estimate of the channel impulse response (CIR) has been obtained, the equalization can be performed using an existing ZF equalizer by taking the FFT of the CIR estimate.

This approach to OFDM channel estimation mandates the use a complementary codeset since there are no individual finite-length sequences that have an impulse ACF with an acceptable peak-to-average power ratio (PAPR). In previous work, evolutionary algorithms (EAs), also referred to as genetic algorithms (GAs), have proven effective in optimizing arbitrary criteria [4]. For example, EAs have been used to find OFDM synchronization sequences with minimal ACF sidelobes [5]. In this paper, we use an EA to find a channel estimation sequence that is complementary to an existing preamble synchronization sequence.

Although this work is relevant to many OFDM systems, including IEEE 802.11a and $802.11 \mathrm{~g}$, we focus on MBOFDM, the first ultra-wideband (UWB) technology to obtain international standardization [6]. MB-OFDM is representative of contemporary OFDM wireless systems in that it supports several data rates. This flexibility allows communication over 


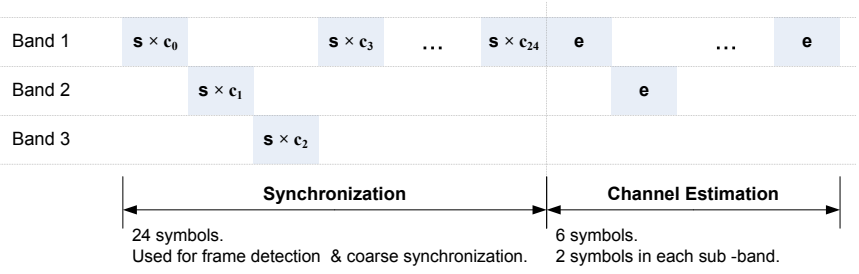

Fig. 2. Preamble sequence in MB-OFDM.

separations of less than $10 \mathrm{~cm}$ to well over $10 \mathrm{~m}$ and leads to significant variation in channel conditions. With longer UWB channels susceptible to significant frequency-selective fading, it is necessary that the channel estimation is robust and accurate. At the same time, given that an MB-OFDM receiver operates at at least 528MSPS, the total computational complexity must be kept to an absolute minimum in order for cost-effective digital hardware to be realizable.

In recognition of the variety of UWB channel conditions, the IEEE 802.15.3a working group defined 4 UWB channel models [7], denoted as CM1 through CM4, to model lineof-sight (LOS) and non-line-of-sight (NLOS) environments possessing average RMS delay spreads of $5 \mathrm{~ns}$ to $25 \mathrm{~ns}$. These channel models all use a parameterized Salem-Venezuela (SV) model. In this paper, we use these channel models to show how the proposed equalization is effective in both short and long LOS and NLOS channels.

We begin by describing how we will use complementary codesets for channel estimation in Section II. Then, in Section III, we present an EA that, based on a predefined preamble synchronization sequence, searches for a complementary channel estimation sequence with a given PAPR. The results of Section IV then quantify end-to-end packet error rate (PER) and give an example of several high-performing channel estimation sequences appropriate for MB-OFDM. Finally, we present our conclusions in Section V.

\section{COMPLementary CODESETS}

Consider the 30 symbol MB-OFDM preamble shown in Fig. 2. The first 24 symbols are based on a 128 chip synchronization sequence $s(n)$. The receiver uses the auto-correlation of this sequence for frame detection and symbol synchronization as well as potentially frequency and sampling offset estimation. Immediately following this, the next 6 symbols are based on a 128 chip channel estimation sequence $e(n)$. Also shown in a 24 chip cover sequence $c_{k}$. This cover sequence has no impact on the ACF and is used only to delineate the synchronization and channel estimation stages of the preamble. Note that, depending on the selected time-frequency code (TFC), there may be frequency hopping. This is of little impact on the channel estimation presented in this paper since it is assumed that each sub-band is estimated independently.

We denote the ACF of the original synchronization sequence, obtained from the MB-OFDM standard [6], as $\phi_{s s}(n)$ and the ACF of our a new ideal complementary channel estimation sequence as $\phi_{e e}(n)$. Our objective is therefore to obtain a channel estimation sequence such that

$$
\phi_{s s}(n)+\phi_{e e}(n)=2 \delta(n)
$$

where $\delta(n)$ denotes an impulse function where $\delta(0)=1$ and $\delta(n)=0$ for $n \neq 0$. We can rearrange (1) to conclude that ideal channel estimation sequence must have an ACF $\phi_{e e}(n)=-\phi_{s s}(n)$ for $n>0$.

MB-OFDM is different from many other OFDM systems in that it uses a 37 sample zero-pad (ZP) rather than the more conventional cyclic prefix (CP). In addition, the frequency hopping used by some TFCs can potentially completely eliminate inter-block interference (IBI). For both these reasons, it is necessary to base all ACFs on linear convolution instead of circular convolution. Although we do not explicitly consider systems other than MB-OFDM, we note that using a $\mathrm{CP}$ will require modification to (2).

Using linear convolution, we denote the ACF of the soughtafter channel estimation sequence as

$$
\phi_{e e}(n)=\sum_{m=n}^{N-1} e(m) e(m-n)
$$

Since the $n^{\text {th }}$ tap of this ACF is the sum of $N-n$ coefficients, it is necessary to limit evaluation of the fitness of the ACF to a region-of-interest (ROI) of $0 \leq n<L$, where $L$ denotes channel maximum delay spread. It is important that $L<<N$, such as $L=64$ in a $N=128 \mathrm{MB}-\mathrm{OFDM}$ system, so that there are sufficient degrees of freedom in the code-space.

To balance computational complexity against performance, we restrict the candidate channel estimation sequences to being real-valued at the transmitter and binary at the receiver. In other words, we can denote the ACF of practical sequences as

$$
\phi_{\hat{e} \hat{e}}(n)=\sum_{m=n}^{N-1} \hat{e}(m) \operatorname{sgn}[\hat{e}(m-n)]
$$

Although this simplification has negligible impact on performance, it significantly reduces complexity since the $N$ multiplications used in the receiver correlation can be simplified to $N$ additions/subtractions.

Fig. 3 shows the relevant normalized ACFs for TFC 1 of the MB-OFDM standard. Note that the ACF at $n=0$ is not shown since the normalization forces it to 1 . In this figure, the maximum channel delay was set to $L=64$. We can see that the practical ACF, obtained using the EA of Section III, is almost ideal when $n<L$ and totally useless when $n \geq L$. Since an OFDM system assumes that the majority of channel energy arrives within the $\mathrm{ZP}$ or $\mathrm{CP}$, lest IBI cripple performance, this causes little or no loss so long as $L$ exceeds the ZP or CP. We conclude from Fig. 3 that practical complementary codesets can be used to obtain an almost ideal ACF.

\section{EvOlutionary SEARCH}

Finding a finite-length sequence possessing a specific ACF can require the searching of a very large code space. To make this problem computationally feasible, we employ the 


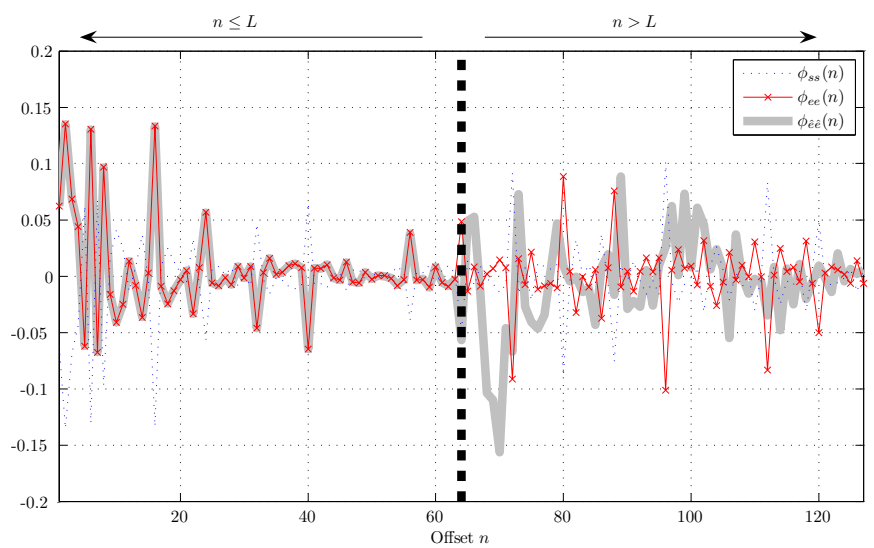

Fig. 3. Comparison of auto-correlations $\phi_{s s}(n), \phi_{e e}(n)$ and $\phi_{\hat{e} e \hat{e}}(n)$.

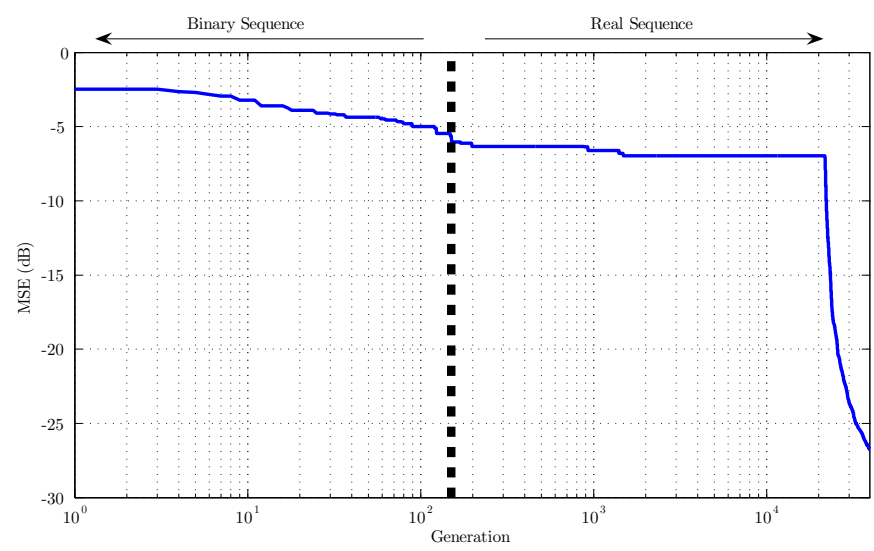

Fig. 4. MSE of population's best chromosome vs. generation count.

Even through we are seeking real-valued sequences, the PAPR

$$
\operatorname{PAPR}_{\hat{e}}=\frac{\max _{0 \leq n<N} \hat{e}^{2}(n)}{\operatorname{mean}_{0 \leq n<N} \hat{e}^{2}(n)}
$$

of the estimation sequence must not exceed the capability of the transceiver. The EA considers this limit by repeating the mutation until the PAPRs of all sequences are within the limit $\mathrm{PAPR}_{\mathrm{MAX}}=10$. We contend that this value is reasonable for two reasons. First is because the OFDM data symbols have a higher worst-case PAPR of $N=128$. Second is due to ease with which transmitter non-linearities can be mitigated through pre-distortion of the channel estimation sequence.

To help our search for real-valued sequences avoid localminima, we define two distinct phases of mutation. The first phase limits the sequences $\hat{e}(m)$ to $\{+1,-1\}$ and defines mutation as the inversion of the relevant chips. This phase ends when the best chromosome in the population has been stable for several generations. In the second phase, the binary restriction on the transmitted sequence is relaxed in accordance with (3). During this phase, each mutated chip is selected from a Gaussian distribution with unity variance. The mutation for each sequence is repeated until the PAPR of the sequence is less than PAPR $\mathrm{MAX}$.

Fig. 4 shows how the MSE, defined as $\min _{\forall p} \frac{1}{F_{p}^{\prime}}$, improves with each generation. For all results in this paper, the population size is $P=100$ and the chip mutation probability is $\alpha=0.01$. The fitness sharing is parameterized with $\sigma=20$ and $\kappa=4$. The termination condition is that the MSE is less than $-45 \mathrm{~dB}$. This condition was selected so that the error resultant from the non-ideal ACF is less than the receiver's thermal noise.

\section{REsults}

The MB-OFDM standard defines seven synchronization sequences such that each TFC has a unique preamble. In this section, we provide seven channel estimation sequences that are complementary to their respective synchronization sequences. We also quantify the potential PER improvements

$$
\begin{aligned}
& \Gamma_{k, p}= \begin{cases}1-\left[\frac{\left\|\hat{e}_{k}-\hat{e}_{p}\right\|}{\sigma}\right]^{\kappa}, & \text { when }\left\|\hat{e}_{k}-\hat{e}_{p}\right\|<\sigma \\
0, & \text { otherwise }\end{cases} \\
& \text { where }\left\|\hat{e}_{k}-\hat{e}_{p}\right\| \text { denotes the Euclidean distance between } \\
& \text { sequences } \hat{e}_{k} \text { and } \hat{e}_{p} . \sigma \text { and } \kappa \text { are arbitrary real constants. }
\end{aligned}
$$



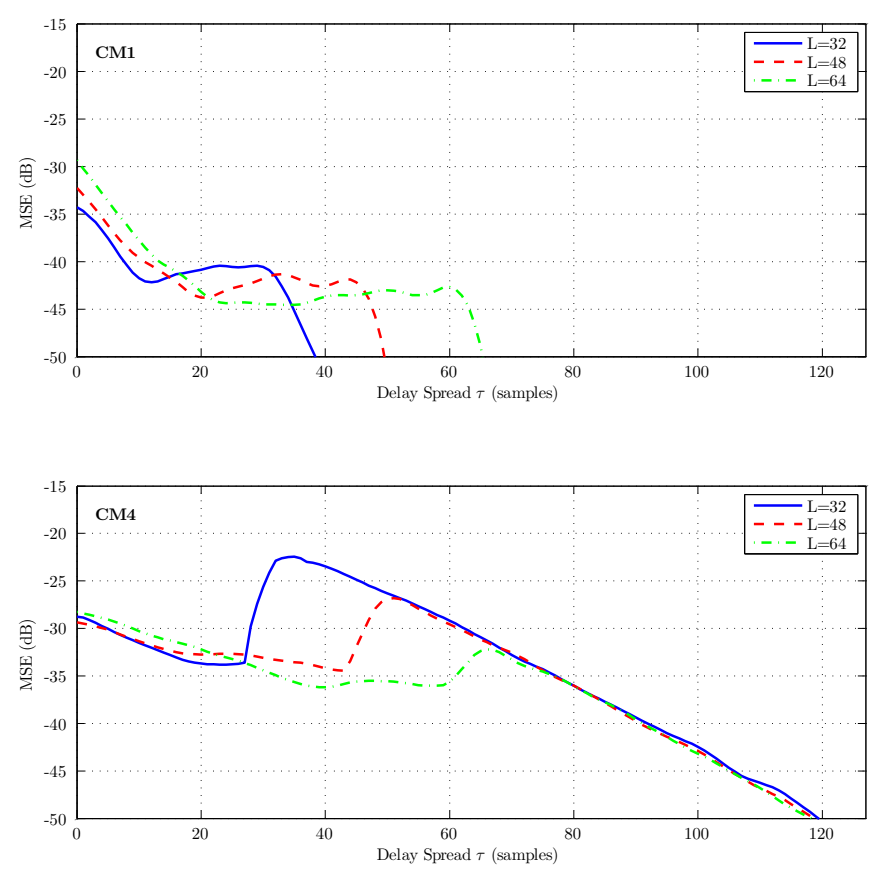

Fig. 5. MSE in channel estimation with respect to delay spread $\tau$.

that can be achieved by employing this approach to channel estimation.

\section{A. ACF Region-of-Interest}

It is important that the ACF ROI $L$ is appropriate for the expected channel delay spread. If $L$ is too small, the ACF of (1) will have significant sidelobes at noticeable delays and will introduce error into the channel estimation. Conversely, if $L$ is too large, the accuracy of dominant lower taps of the ACF will be degraded in favor of reducing fringe sidelobes that will never be encountered. We must therefore find an $L$ for which the error due to the non-ideal channel estimation sequence is balanced with the energy lost due to truncation of the CIR.

To determine $L$, Fig. 5 shows the MSE of the estimated CIR as a function of channel delay for ACF ROIs of 32, 48 and 64 samples. Note that each channel estimation sequence was obtained via Alg. 1 and that both LOS and NLOS channels are considered via $\mathrm{CM} 1$ and $\mathrm{CM} 4$ respectively. We observe that CM1 channels lose little energy even under truncations as aggressive as $L=32$. This is not the case in CM4 channels where the ROI must be larger to avoid spikes in the MSE. We conclude that an $L=64 \mathrm{ROI}$ is an effective compromise. Although this does lead to a slightly higher MSE for taps $n>30$ in CM1, the discarded energy is so small that it is of little consequence.

\section{B. Channel Estimation Error}

Fig. 6 compares the MSE of FDE and TDE in both CM1 and CM4 at a range of SNRs. The ZF FDE channel estimation is performed by calculating the impact of the channel on the magnitude and phase of symbol's $N$ subcarriers. Although this estimation is computationally simple, it does not consider any
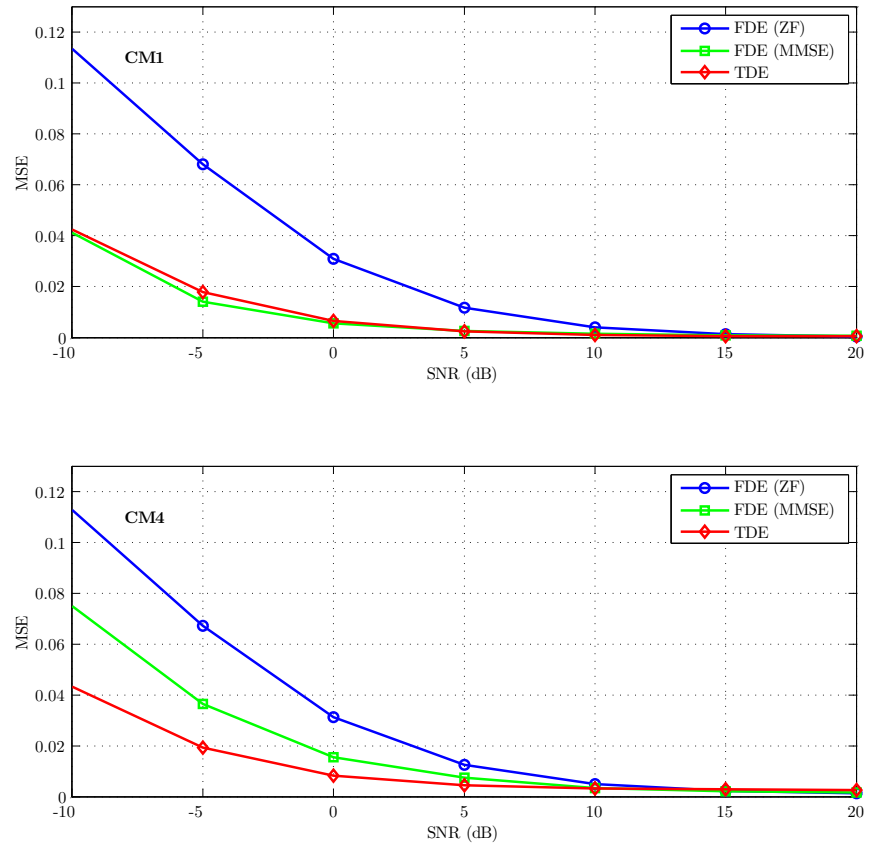

Fig. 6. MSE in channel estimation.

correlation that may exist between subcarriers. This makes it perform poorly in short CM1 channels.

Minimum mean square error (MMSE) FDE [10] uses a pre-computed estimate of the degree of subcarrier correlation to improve the initial ZF estimate. Although this does significantly reduce the MSE, it is extremely computationally intensive as it relies on implementing a long finite-impulse response (FIR) filter with complex coefficients.

The TDE techniques proposed in this paper offer performance that is at least as good as MMSE estimation but at a fraction of the computational complexity. In other words, since the correlator hardware is reused from the synchronization stage, the complexity of the TDE is almost equivalent to that of ZF FDE. In addition, since MMSE FDE requires off-line statistical modeling to calculate the expected channel autocovariance matrices, TDE actually outperforms MMSE FDE in long channels that are difficult to characterize.

\section{Packet Error Rate}

Fig. 7 and Fig. 8 show the PER of the proposed equalization scheme for low and high rates respectively in CM1. Fig. 9 and Fig. 10 show the same data rates in CM4. It can be seen that TDE can outperform ZF FDE by over $1 \mathrm{~dB}$. Although MMSE FDE can achieve PER performance similar to TDE, its tremendous computational complexity makes it infeasible in practical receivers.

\section{Optimal Channel Estimation Sequences}

Effective channel estimation sequences for all seven TFCs in MB-OFDM are provided in Table I. These sequences were obtained via the evolutionary algorithm of Section III and the TFC 1 sequence was used in the PER simulations. 
(a) Samples $1 \ldots 64$

\begin{tabular}{|c|c|c|c|c|c|c|c|}
\hline & TFC 1 & TFC 2 & TFC 3 & TFC 4 & TFC 5 & TFC 6 & TFC 7 \\
\hline 1 & 3.0967 & -0.0113 & 1.6018 & 0.7410 & 0.0056 & 1.9453 & -0.3981 \\
\hline 2 & -0.0076 & -0.0076 & 1.0000 & 0.2811 & 0.0019 & -1.4676 & 0.0232 \\
\hline 3 & 0.0311 & 1.4329 & -0.6233 & 0.5020 & 0.0022 & 1.6289 & -1.0143 \\
\hline 4 & 1.4040 & -2.1373 & 2.0245 & -2.4111 & 1.6342 & -0.1946 & 0.5150 \\
\hline 5 & -0.0059 & 0.0734 & 0.0568 & 0.7938 & -0.0010 & 0.4971 & -1.0225 \\
\hline 6 & 1.0723 & -0.0003 & -0.9004 & -0.8126 & 1.7735 & 2.2154 & -1.1657 \\
\hline 7 & 0.1597 & -1.0218 & -0.0116 & 0.7967 & 0.7140 & 0.0227 & -2.3474 \\
\hline 8 & -0.1714 & -0.0335 & 0.8219 & -0.1430 & 0.0034 & -1.4017 & -1.0281 \\
\hline 9 & -1.5204 & 0.8189 & 1.2646 & 2.5625 & -1.9621 & 0.6027 & -0.9118 \\
\hline 10 & -1.9859 & -2.0216 & 0.6737 & -1.5280 & 1.1517 & -0.5410 & -1.0617 \\
\hline 11 & 1.1617 & 2.3473 & -2.3353 & -1.3864 & 0.9414 & 0.8444 & -1.2883 \\
\hline 12 & -0.0011 & 0.6383 & 1.0708 & -0.6797 & -0.0047 & -0.9182 & -0.7165 \\
\hline 13 & 1.7849 & 0.5407 & -1.5883 & 0.0120 & -0.0025 & -1.1306 & -2.2673 \\
\hline 14 & 0.4617 & 0.9001 & 0.7420 & -2.6034 & -0.0017 & -0.4401 & -0.3438 \\
\hline 15 & 0.6386 & -1.1154 & 0.6738 & 0.4725 & 0.8390 & 1.6879 & 0.5741 \\
\hline 16 & -2.0351 & -0.6311 & 1.9906 & -0.3799 & 1.1587 & 1.4459 & 2.4739 \\
\hline 17 & 1.1948 & -0.3380 & -0.4801 & -2.0178 & -1.4201 & 1.1615 & -1.0220 \\
\hline 18 & -1.5833 & 1.2513 & -1.5613 & 1.2552 & 0.4610 & -1.0463 & 1.0169 \\
\hline 19 & 1.4779 & -0.8851 & -1.0937 & -1.0007 & -0.0111 & -0.3104 & 1.4586 \\
\hline 20 & 1.5136 & 0.6919 & -1.2467 & 0.9643 & 2.6271 & -0.7841 & -1.1982 \\
\hline 21 & 1.1621 & 1.3744 & 1.5607 & 2.8268 & 2.2129 & -1.1041 & 0.1301 \\
\hline 22 & 1.4769 & 1.0162 & -0.9285 & -2.1326 & 1.9171 & -1.0132 & -1.2833 \\
\hline 23 & 0.0034 & 1.9988 & 0.5692 & 0.8537 & -1.5789 & 1.3988 & -1.1773 \\
\hline 24 & -1.7864 & 1.0241 & -1.2999 & 1.6839 & -1.2704 & -2.1574 & 2.4103 \\
\hline 25 & -1.2172 & -0.7992 & -1.0114 & 0.5800 & -0.6384 & -0.0000 & -1.5466 \\
\hline 26 & -0.7650 & 0.3176 & 0.8367 & -0.8879 & -0.7602 & 0.8925 & 1.0590 \\
\hline 27 & 1.8126 & 0.2414 & -1.6040 & 0.9113 & -1.1909 & -1.5897 & -0.8497 \\
\hline 28 & -1.5593 & 0.9585 & -0.2797 & -1.6924 & 0.5303 & 1.4649 & 1.0553 \\
\hline 29 & 0.4103 & 0.9278 & -0.5020 & -0.6630 & 0.2454 & 0.5664 & -0.8616 \\
\hline 30 & 1.6778 & -0.7228 & 1.2064 & 0.9468 & 1.3207 & 1.6169 & -0.9315 \\
\hline 31 & 1.3377 & 1.2293 & 1.4782 & 0.9456 & -0.6480 & 1.0912 & -0.6684 \\
\hline 32 & 1.4785 & 1.4171 & 1.4924 & -0.2491 & 1.0924 & -1.8913 & 1.8057 \\
\hline 33 & -1.0434 & -1.4698 & -1.4157 & -0.1044 & 1.2461 & 1.0533 & -1.1490 \\
\hline 34 & -0.9313 & 0.7233 & -0.8821 & 1.1603 & -1.0636 & -1.1090 & -1.5624 \\
\hline 35 & 0.6725 & 1.1684 & -0.3149 & 1.3497 & 0.2857 & 0.8091 & 0.9787 \\
\hline 36 & 0.1152 & 1.3487 & 0.3495 & -0.9852 & 1.4859 & -1.2121 & -1.2162 \\
\hline 37 & 1.6085 & -0.4606 & -0.2649 & -1.0407 & -0.4482 & 0.0073 & -0.5072 \\
\hline 38 & 2.1369 & -0.7118 & 1.1599 & -0.9219 & 0.8815 & 1.0422 & 0.8772 \\
\hline 39 & 0.9745 & -0.8256 & -0.0052 & 1.0253 & 0.3178 & -1.3392 & -0.8684 \\
\hline 40 & 0.2796 & -1.0172 & -0.8126 & 0.8328 & 1.3256 & -1.1169 & -1.0780 \\
\hline 41 & -0.7421 & -1.0549 & -0.9072 & 1.2253 & 1.1138 & -0.4611 & -0.7848 \\
\hline 42 & -0.3172 & -0.1350 & 0.9960 & -1.5046 & 1.6187 & 0.9210 & 0.0115 \\
\hline 43 & -0.5981 & -0.0532 & 1.2655 & -0.6487 & -0.2972 & -1.0844 & -0.4779 \\
\hline 44 & -0.0141 & 0.1984 & -0.7820 & 0.8832 & 0.2805 & -1.6001 & -0.5622 \\
\hline 45 & -1.0332 & -1.5916 & -1.0784 & 0.6158 & -1.1082 & 0.1250 & 1.0522 \\
\hline 46 & -1.5122 & -1.3496 & 0.5885 & 0.8076 & 1.0709 & 1.0864 & -0.7957 \\
\hline 47 & -2.0664 & -1.4318 & 0.9557 & 0.6395 & -1.2515 & -1.1813 & -0.4366 \\
\hline 48 & -0.7741 & 0.8070 & -1.1762 & 1.2472 & 0.5310 & 1.1007 & -0.9666 \\
\hline 49 & -0.5790 & 1.0265 & 0.7644 & -0.8077 & 0.3126 & -0.0027 & -0.8695 \\
\hline 50 & -0.0037 & 1.0359 & 0.9470 & 0.5916 & -0.5851 & 0.7934 & 0.7351 \\
\hline 51 & 1.2656 & -0.9555 & 1.0197 & -1.8758 & 0.0126 & -0.9292 & 0.1952 \\
\hline 52 & 0.8974 & 1.2953 & 0.8746 & 0.9067 & 1.0585 & 0.9616 & 0.9691 \\
\hline 53 & 0.6514 & -0.9777 & 1.0478 & 1.0713 & -0.5819 & 0.7209 & -1.2858 \\
\hline 54 & 0.9544 & -0.1112 & -0.9992 & 1.4011 & -0.0060 & 1.2180 & -1.8018 \\
\hline 55 & 1.4149 & 0.9402 & 0.8257 & 1.1650 & -0.5180 & 0.9023 & 0.8531 \\
\hline 56 & 1.0804 & 0.3469 & 1.0224 & 0.1600 & -1.0509 & 0.7551 & 0.9848 \\
\hline 57 & 1.4089 & -1.0547 & -1.0124 & 1.4590 & 0.8360 & -0.3993 & -1.1119 \\
\hline 58 & -1.0598 & -0.0305 & -0.0883 & -1.3642 & -1.2564 & -1.0881 & 0.3743 \\
\hline 59 & 1.6441 & -1.0383 & 1.0014 & 1.1983 & 1.5468 & 0.6921 & -0.4241 \\
\hline 60 & 0.9501 & -1.1384 & -0.4994 & 0.0379 & 0.5591 & 0.6548 & -1.0046 \\
\hline 61 & 0.9754 & 0.2246 & 1.6335 & 0.0032 & -1.1545 & -0.4220 & -0.9938 \\
\hline 62 & -0.2760 & 1.4309 & -0.5987 & 1.2300 & 0.8527 & -1.0995 & -1.0023 \\
\hline 63 & -0.3367 & 0.8507 & -1.2152 & -0.2162 & 0.8350 & 1.1992 & 0.9859 \\
\hline 64 & 1.1239 & 0.5341 & -0.9050 & -0.7915 & -1.7449 & 1.2929 & -0.0817 \\
\hline
\end{tabular}

(c) Samples $65 \ldots 128$

\begin{tabular}{|c|c|c|c|c|c|c|c|}
\hline & TFC 1 & TFC 2 & TFC 3 & TFC 4 & TFC 5 & TFC 6 & TFC 7 \\
\hline 65 & 1.4992 & 1.5937 & -1.0075 & 0.5084 & 1.5503 & 0.7981 & 0.9814 \\
\hline 66 & 0.4854 & 1.0062 & 0.1894 & 1.0154 & 1.0666 & 0.5292 & 0.8174 \\
\hline 67 & 1.2874 & -0.9281 & -1.0289 & 1.3578 & 0.6652 & 0.1974 & 1.0127 \\
\hline 68 & -0.4520 & 1.0104 & 0.4700 & 0.2713 & 0.4706 & -1.0897 & -1.4155 \\
\hline 69 & -1.1701 & -0.6125 & 0.9388 & -1.0593 & -0.9341 & 0.0135 & 1.6613 \\
\hline 70 & 1.5227 & -1.1338 & -1.1251 & 0.5419 & -1.2420 & 0.6515 & 0.8913 \\
\hline 71 & -0.3317 & 0.4576 & 1.0389 & -0.9772 & 1.6277 & -0.7518 & -0.4097 \\
\hline 72 & -1.1454 & -0.3547 & 1.0058 & 1.5766 & 0.6344 & 1.0552 & -1.5819 \\
\hline 73 & 0.9899 & -1.7471 & -0.8249 & 1.2299 & -0.7574 & 1.2369 & -0.0044 \\
\hline 74 & -1.0312 & 1.0562 & 0.4682 & 1.2882 & 0.0812 & 0.9845 & 1.3727 \\
\hline 75 & -2.1262 & -1.5252 & -0.8591 & 0.7677 & 0.4460 & -1.3633 & 1.0698 \\
\hline 76 & 1.1406 & 1.0751 & -0.6382 & 1.3442 & -0.8786 & 0.9948 & 1.0345 \\
\hline 77 & -1.0091 & 0.7283 & -0.9838 & 0.9956 & -0.0058 & 0.6011 & 1.8009 \\
\hline 78 & -0.7607 & 1.2378 & -0.6055 & 0.1404 & 0.9070 & 1.2722 & -1.1836 \\
\hline 79 & 1.1905 & -1.1167 & -0.8925 & 0.4517 & 0.4027 & 0.9464 & 1.3130 \\
\hline 80 & -1.2160 & 0.0005 & 0.2153 & -0.8706 & -0.9291 & 1.3169 & 0.1968 \\
\hline 81 & 0.9445 & 1.1348 & -0.8190 & -1.0266 & -0.7664 & 0.4654 & -0.1400 \\
\hline 82 & 0.0024 & 0.8206 & -0.8684 & 0.6744 & 0.3855 & 1.1951 & -0.8169 \\
\hline 83 & -0.7934 & 0.8250 & -0.8798 & -1.1740 & -1.2241 & 1.1067 & 1.0822 \\
\hline 84 & -0.8948 & 0.1262 & -1.0895 & 0.0685 & -1.1838 & 0.9938 & 0.6661 \\
\hline 85 & 0.4475 & -0.6099 & 0.9178 & 0.0869 & 0.4685 & 1.4398 & 0.6356 \\
\hline 86 & -1.5636 & 0.9098 & 0.9841 & -0.5220 & 1.1926 & 0.8887 & -1.0385 \\
\hline 87 & 0.3876 & 0.4728 & 1.3279 & 0.7571 & -1.4378 & -1.2228 & 0.0630 \\
\hline 88 & -1.0356 & 1.8201 & -1.5777 & -0.9041 & 0.3874 & 0.7793 & 0.7943 \\
\hline 89 & -1.0469 & -0.7564 & -0.0954 & 1.0282 & -1.1871 & -0.7677 & 0.9187 \\
\hline 90 & -0.5597 & 1.0256 & -1.3685 & 1.4686 & -1.0848 & 0.3436 & 0.9083 \\
\hline 91 & -0.3670 & -1.1906 & -1.1945 & -0.1371 & 0.0015 & -0.5336 & -0.4480 \\
\hline 92 & -1.0396 & 0.3965 & -1.4513 & -0.9481 & -0.2429 & 0.0006 & 0.5887 \\
\hline 93 & 0.8715 & -0.2978 & -1.0479 & -1.0571 & 1.3195 & 1.0520 & -0.9111 \\
\hline 94 & -1.9810 & 0.5952 & -0.8051 & -0.2364 & 1.6051 & 0.9904 & -0.5643 \\
\hline 95 & -0.8104 & -0.7957 & 0.9790 & -0.9443 & 0.7856 & -1.1090 & 0.7892 \\
\hline 96 & -1.0481 & 0.7412 & -1.3610 & -1.2709 & -1.2594 & -0.3923 & -0.9977 \\
\hline 97 & -0.7492 & -0.4459 & 0.8712 & 0.8444 & -1.5789 & -0.3564 & -0.9166 \\
\hline 98 & 0.8818 & 0.2438 & -0.7655 & -0.7990 & -1.0629 & -0.8142 & -0.5766 \\
\hline 99 & -1.4309 & 1.3266 & 0.9829 & -1.2177 & -1.3412 & -0.3976 & 1.3941 \\
\hline 100 & 1.0485 & -0.6089 & -1.2158 & -1.8743 & 0.5179 & -1.4359 & -0.9883 \\
\hline 101 & -2.0045 & 1.3896 & -1.6772 & 0.9054 & -0.8890 & -1.0530 & -1.6224 \\
\hline 102 & -0.0026 & 0.0390 & -0.8467 & -1.3045 & -0.5164 & 0.9891 & 0.9890 \\
\hline 103 & -0.7028 & 1.0958 & -0.2180 & 1.0311 & 0.3972 & -1.3110 & -1.8990 \\
\hline 104 & -0.7051 & 0.8693 & -0.0901 & -1.0074 & 1.1317 & -1.1253 & -1.3677 \\
\hline 105 & -0.8922 & 0.9774 & 0.9071 & 0.6766 & -1.4413 & 0.0027 & -0.2703 \\
\hline 106 & -1.0133 & 0.6492 & -1.5684 & 1.1088 & 0.2985 & -0.4740 & -0.9527 \\
\hline 107 & -1.0113 & 0.7672 & -0.5969 & -0.8933 & -1.4271 & -0.3351 & 1.0078 \\
\hline 108 & 0.8678 & -0.6722 & -0.8482 & 0.9879 & 0.0012 & 0.7481 & -0.5318 \\
\hline 109 & 0.9814 & 0.7062 & -0.7216 & -0.9640 & -0.9384 & -0.6180 & -1.5952 \\
\hline 110 & -0.6137 & 0.7765 & -0.9043 & 1.0768 & -1.0870 & -0.8800 & 0.2702 \\
\hline 111 & 0.6292 & 1.0398 & 1.0679 & -0.9491 & 0.9846 & 1.1281 & 0.3419 \\
\hline 112 & 0.7830 & 0.9733 & -1.0150 & 0.9571 & -1.5938 & 1.0349 & -0.7192 \\
\hline 113 & -0.7868 & 1.0191 & 1.1588 & 1.0451 & 1.4767 & 1.0109 & 0.9883 \\
\hline 114 & -0.4964 & -1.3029 & -1.0058 & 1.4849 & -1.2787 & 1.1518 & -0.0418 \\
\hline 115 & -1.1238 & -0.9675 & 0.7411 & -0.3374 & 0.3830 & 1.1300 & 0.8512 \\
\hline 116 & 1.3608 & -1.0485 & -1.5607 & 0.8711 & 0.9930 & -0.9575 & -0.3651 \\
\hline 117 & -1.8799 & 1.0713 & 0.6698 & -0.7731 & 0.8245 & 1.0257 & 1.0776 \\
\hline 118 & 0.7429 & -0.9991 & -0.9241 & 1.0932 & 0.6973 & 0.9326 & -0.9511 \\
\hline 119 & -0.5944 & -1.1159 & 0.9405 & -0.9855 & -0.6812 & -1.4521 & 0.8588 \\
\hline 120 & 1.2252 & -0.8415 & 0.1291 & 1.0658 & 0.7708 & 1.0707 & 0.3803 \\
\hline 121 & 0.9940 & 1.2548 & 1.1897 & 0.9757 & 1.0660 & -0.6667 & -0.9768 \\
\hline 122 & 1.1687 & 0.8708 & 0.6988 & 0.8954 & 0.8528 & -0.9725 & 1.0348 \\
\hline 123 & -0.3805 & 0.6727 & -1.0221 & 0.8433 & 1.0237 & 0.2983 & 1.2738 \\
\hline 124 & 0.9386 & 0.8460 & 1.1414 & 0.2457 & -1.0883 & -0.7621 & -0.9863 \\
\hline 125 & -0.7517 & -0.8159 & -1.0696 & -1.2666 & 1.0869 & 1.2078 & 0.8017 \\
\hline 126 & -1.0210 & -1.2087 & 1.2031 & -0.5198 & 0.2239 & 0.6378 & -1.5032 \\
\hline 127 & 0.0039 & 0.5211 & 0.8950 & -1.1812 & 0.1716 & -1.5741 & -1.5416 \\
\hline 128 & 1.2494 & -0.3989 & -0.1580 & -0.8098 & 0.7164 & 1.0775 & 0.7686 \\
\hline
\end{tabular}

TABLE I

EFFective Channel Estimation Sequences For MB-OFDM.

We conclude that even though TDE requires modification to the existing MB-OFDM standard, its significant performance improvements make it particularly attractive as a proprietary differentiator. We also note that since the synchronization sequence remains unchanged, the use of TDE would not impact legacy receivers in adjacent networks.

\section{CONCLUSION}

We have presented a novel approach to time-domain channel estimation for MB-OFDM systems that uses a complementary codeset to achieve a near-ideal ACF. We showed how an EA can use the preexisting preamble synchronization sequences to find complementary real-valued channel estimation sequences that facilitate efficient and accurate receiver equalization. We 


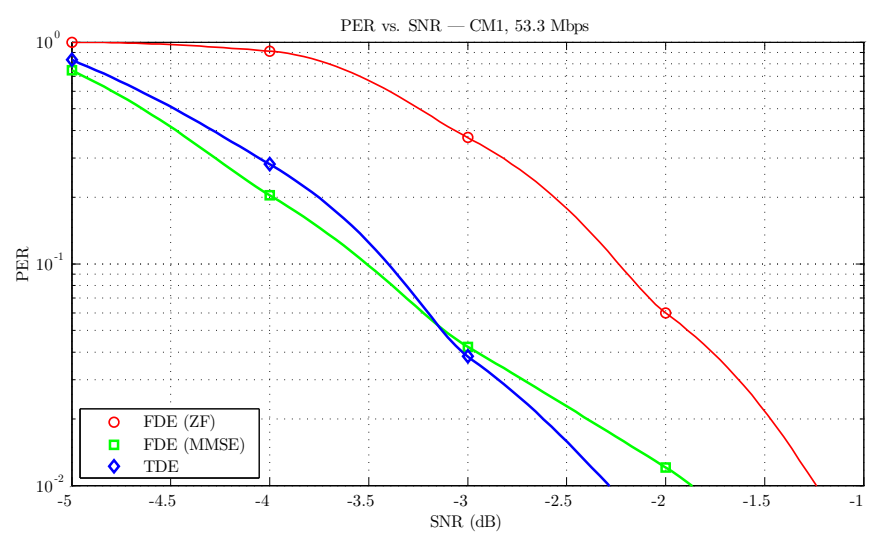

Fig. 7. PER at $53.3 \mathrm{Mbps}$ for FDE and TDE for CM1.

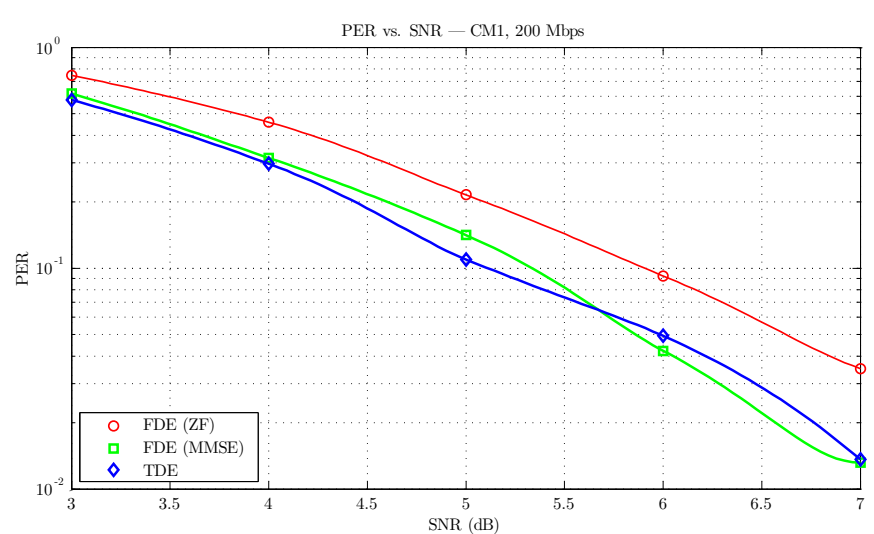

Fig. 8. PER at $200 \mathrm{Mbps}$ for FDE and TDE for CM1.

provided example channel estimation sequences for all 7 TFCs and demonstrated PER improvements up to $1 \mathrm{~dB}$ with Monte Carlo simulations.

Future work will develop a multi-objective evolutionary algorithm that aims to derive entirely new synchronization and estimation sequences that offer an optimal balance between $\mathrm{ACF}$, cross-correlation function, PAPR and quality of channel estimation.

\section{REFERENCES}

[1] Z. Wang and G. B. Giannakis, "Wireless multicarrier communications," IEEE Signal Processing Magazine, vol. 17, no. 3, pp. 29-48, 2000.

[2] M. Luise, R. Reggiannini, and G. M. Vitetta, "Blind equalization/detection for OFDM signals over frequency-selective channels," IEEE Journal on Selected Areas in Communications, vol. 16, no. 8 , pp. $1568-1578$, Oct. 1998.

[3] A. Batra, J. Balakrishnan, G. R. Aiello, J. R. Foerster, and A. Dabak, "Design of a multiband OFDM system for realistic UWB channel environments," in IEEE Transactions on Microwave Theory and Techniques, vol. 52, no. 9, Sept. 2004, pp. 2123-2138.

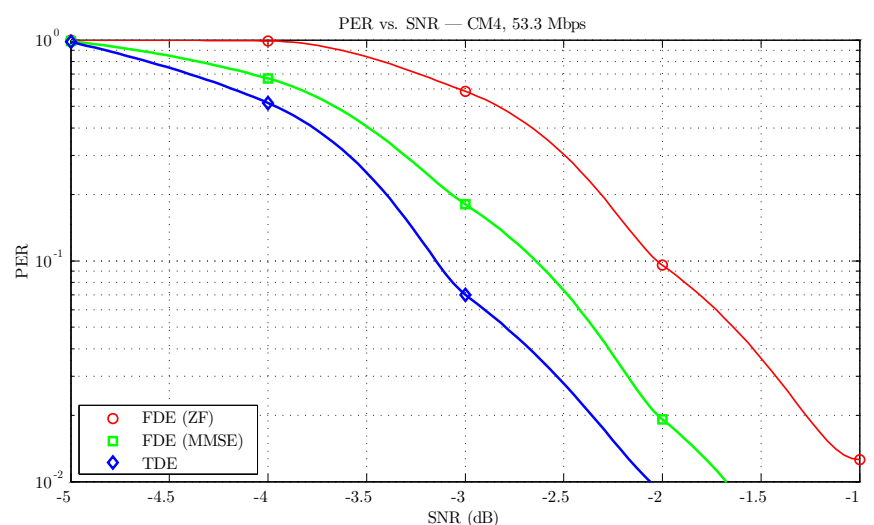

Fig. 9. PER at 53.3 Mbps for FDE and TDE for CM4.

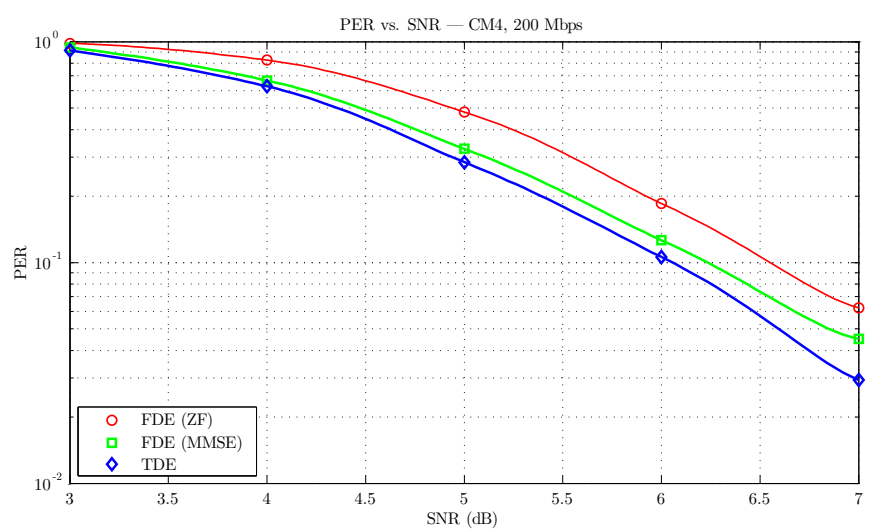

Fig. 10. PER at 200 Mbps for FDE and TDE for CM4.

[4] H. Dong, B. Wang, C. Gu, and G. Feng, "Synthesis of binary sequences with good auto- and cross-correlation properties by GA," in Proceedings of International Conference on Communication Technology, Oct. 1998 p. 5.

[5] O. Ureten, S. Tascioglu, N. Serinken, and M. Yilmaz, "Search for OFDM synchronization waveforms with good aperiodic autocorrelations," in Proceedings of CCECE, May 2004, pp. 13-18.

[6] High Rate Ultra Wideband PHY and MAC Standard, ECMA International ECMA-368, Dec. 2005.

[7] A. F. Molisch, J. R. Foerester, and M. Pendergrass, "Channel models for ultrawideband personal area networks," IEEE Wireless Commun. Mag., pp. 14-21, Dec. 2003.

[8] B. Natarajan, S. Das, and D. Stevens, "An evolutionary approach to designing complex spreading codes for DS-CDMA," IEEE Transactions on Wireless Communications, no. 5, pp. 2051-2056, Sept. 2005.

[9] C. Fonseca and P. Fleming, "An overview of evolutionary algorithms in multiobjective optimization," Evol. Computing, no. 1, pp. 1-16, 1995.

[10] J. J. van de Beek, O. Edfors, M. Sandell, S. K. Wilson, and P. O. Brjesson, "On channel estimation in OFDM systems," in Proceedings of the IEEE Vehicular Technology Conference, July 1995, pp. 815-819. 